\title{
El efecto del laboratorio en el aprovechamiento de los estudiantes del curso Métodos Cuantitativos para Administración de Empresas I
}

\author{
Wanda Velázquez Rosado|wanda.velazquez@upr.edu \\ Universidad de Puerto Rico, Recinto de Río Piedras \\ Wanda Villafañe Cepeda | wanda.villafane1@upr.edu \\ Universidad de Puerto Rico, Recinto de Río Piedras \\ José C.Vega Vilca | jose.vega23@upr.edu \\ Universidad de Puerto Rico, Recinto de Río Piedras
}

Recibido en: 28 de octubre de 2014

Aceptado en: 19 de mayo de 2015

\section{RESUMEN}

Este trabajo investigó el efecto de los laboratorios en el aprovechamiento de los estudiantes en el curso Métodos Cuantitativos para Administración de Empresas I. La literatura indica que el uso de laboratorios en distintas clases, incluyendo la de Matemática, ha tenido un impacto positivo en el aprovechamiento de los estudiantes. Mediante un análisis de covarianza ( $p v a l u e<0.05$ ), se encontró que los promedios del examen de mitad de curso, así como del examen final, fueron mayores entre los estudiantes que asistieron al laboratorio en comparación con los que no asistieron al mismo, lo que demuestra la efectividad de esta estrategia.

Palabras clave: métodos cuantitativos, laboratorios, precálculo, administración de empresas, tecnología

\section{ABSTRACT}

This study investigated the effect of the laboratories in the achievement of the students in the course of Quantitative Methods for Business Administration I. The literature indicates that the use of laboratories in different classes, including Mathematics, has had a positive impact on the achievement of the students. Through an analysis of covariance ( $p$ value $<0.05$ ), it was found that the means of the midterm exam, as well as the final exam, were higher for the students that attended the laboratories when compared to the students who did not attend the laboratories, demonstrating the effectiveness of this strategy.

Keywords: quantitative methods, laboratories, precalculus, business administration, technology 
Las universidades que preparan estudiantes en el área de administración de empresas del mundo requieren que sus egresados tengan competencias cuantitativas. Para cumplir con este objetivo, muchas de las facultades incluyen como parte de su currículo cursos de precálculo y cálculo; no obstante, por años han surgido esfuerzos para reformar el currículo de estas áreas, de modo que suplan las necesidades de otras disciplinas tales como economía y finanzas (Gordon, 2010).

Aún con los esfuerzos realizados para reformar el currículo de Matemática, se sigue encontrando que los estudiantes tienen dificultades en el aprendizaje de esta disciplina (Aponte, Pagán, Quintero, Balet, Nazario, \& Velázquez, 2003; Capraro, Capraro, \& Rupley, 2012; Gordon, 2010; Kachapova \& Kachapov, 2012; National Council of Teacher of Mathematics (NCTM), 2000). Además, se ha encontrado que los estudiantes que aprueban los cursos de Matemática, tienen dificultades en cursos subsecuentes, esto es, no pueden transferir el conocimiento adquirido en sus cursos a otras disciplinas (Gordon, 2010).

En el caso particular de la Universidad de Puerto Rico, Recinto de Río Piedras (UPRRP), se ha encontrado que existe un alto por ciento de fracasos de los estudiantes en los cursos cuantitativos, como lo son precálculo y cálculo. Específicamente, el por ciento de fracasos en el curso Métodos Cuantitativos para Administración de Empresas I (MECU 3031) que ofreció la Facultad de Administración de Empresas (FAE) durante los años académicos 2002-2003 al 2009-2010, se mantuvo en alrededor de un 54\% durante el primer semestre y un $57 \%$ durante el segundo semestre, según muestra la Tabla 1. Se considera fracaso darse de baja, recibir incompleto u obtener una nota de $\mathrm{D}$ o F en el curso. 
El EFECTO DEL LABORATORIO EN EL APROVECHAMIENTO DE LOS ESTUDIANTES...

Tabla 1

Porcentaje de fracasos en el curso MECU 3031 por año académico

\begin{tabular}{lll}
\hline Año académico & Primer semestre & Segundo semestre \\
\hline $2002-2003$ & $\frac{210}{372}=56 \%$ & $\frac{198}{370}=54 \%$ \\
$2003-2004$ & $\frac{248}{425}=58 \%$ & $\frac{241}{414}=58 \%$ \\
$2004-2005$ & $\frac{245}{462}=53 \%$ & $\frac{191}{384}=50 \%$ \\
$2005-2006$ & $\frac{217}{422}=51 \%$ & $\frac{215}{354}=61 \%$ \\
$2006-2007$ & $\frac{235}{462}=51 \%$ & $\frac{152}{244}=62 \%$ \\
$2007-2008$ & $\frac{252}{477}=53 \%$ & $\frac{185}{316}=59 \%$ \\
$2008-2009$ & $\frac{294}{504}=58 \%$ & $\frac{194}{349}=56 \%$ \\
$2009-2010$ & $\frac{208}{368}=57 \%$ \\
\hline
\end{tabular}

$\overline{\text { Fuente: Datos obtenidos de la Oficina de Planificación Académica y Presupuesto de }}$ la Universidad de Puerto Rico, Recinto de Río Piedras.

Algunas organizaciones sugieren que parte de las dificultades que exhiben los estudiantes en el aprendizaje de Matemática se debe a la forma en que se enseña esta disciplina (American Mathematical Association of Two-Year Colleges (AMATYC), 2006; NCTM, 2000, 2005). Estas entidades coinciden en señalar que tradicionalmente el énfasis del currículo de Matemática ha estado dominado por la memorización de hechos y procedimientos aislados. Ante esta situación, sugieren que se cambie el énfasis del currículo a uno que esté dirigido al desarrollo de destrezas con sentido y en contexto, las representaciones y conexiones múltiples, y la solución de problemas.

Varios autores (AMATYC, 2006; Bonham \& Boylan, 2011; Ediger, 2012; Fullmer, 2011; NCTM, 2000, 2005; Wiske, 2004) recomiendan que se usen diversas estrategias para ayudar a los estudiantes a superar las dificultades en Matemática tales como multimedios, solución de problemas, tutorías y laboratorios. Las tecnologías como 
computadoras, correos electrónicos, calculadoras gráficas, internet y recursos multimedios ayudan a que el aprendizaje sea interactivo, colaborativo y enlace con el mundo fuera del salón de clases. Específicamente, Bonham y Boylan (2011) señalan que los programas académicos exitosos utilizan múltiples estrategias de enseñanzaaprendizaje, para mejorar y fortalecer el éxito de los estudiantes en los cursos de Matemática. Mencionan que esto incluye el uso de la tecnología como un suplemento a la instrucción ofrecida en clases y la integración de los laboratorios en la instrucción.

En mayo de 2008, el Senado Académico de la UPRRP aprobó la propuesta de revisión curricular del Bachillerato en Administración de Empresas. En esta propuesta, se especifica que todos los cursos de Métodos Cuantitativos que ofrece la FAE incluirán una hora semanal de laboratorio (de forma voluntaria). Estos laboratorios surgen como respuesta a la situación del alto por ciento de fracasos de los cursos de Métodos Cuantitativos (Senado Académico, 2008). Para justificar esta estrategia, se indica que el estudiante requiere práctica adicional para reforzar destrezas y superar deficiencias que demuestra en los cursos de nivel universitario. Esta estrategia permite además atender al estudiante de una forma más individualizada.

Precisamente, el objetivo principal de este estudio es investigar el impacto de los laboratorios en el aprovechamiento de los estudiantes de todas las concentraciones, en el curso MECU 3031. Ello se determinará comparando las puntuaciones obtenidas en el examen de mitad de curso y el examen final departamental de los estudiantes que asistieron al laboratorio de MECU 3031 versus los que no asistieron.

\section{REVISIÓn DE LITERATURA}

Fullmer (2011) estudió la efectividad de los laboratorios que tomó un grupo de estudiantes en las áreas de lectura, escritura y matemáticas en la Universidad Lincoln, en Pensilvania. Particularmente, en la investigación se consideraron aquellos alumnos que asistieron seis ocasiones o más a dichos laboratorios. Se les administró una preprueba y una posprueba, y se compararon los resul- 
tados de ambas. Los participantes del estudio fueron estudiantes de primer año que estaban matriculados en cursos remediales, porque no cumplían los criterios de admisión a la universidad. Los alumnos asistían a los laboratorios, además de asistir a sus clases regulares de estas materias. En el área de Matemática, los cursos en los que estaban matriculados eran: Álgebra I y Álgebra y sus Aplicaciones. El período de los laboratorios era 50 minutos dos veces por semana, y un total de 25 sesiones. La asistencia al laboratorio tenía un valor del $10 \%$ de la nota del curso. El propósito de los laboratorios era proveer repaso y práctica del contenido que se cubría en los cursos.

$\mathrm{Al}$ inicio del estudio se les administró una preprueba para conocer el nivel de conocimiento en el que se encontraban los estudiantes en los temas del curso. Tomando estos resultados como referencia, se le presentó a cada alumno los problemas que necesitaba aprender. Al final del estudio se administró una posprueba semejante a la preprueba previamente administrada. Se compararon ambos resultados. Para comparar los resultados de la preprueba y la posprueba, se administró una prueba t para muestras pareadas. Se encontró evidencia estadísticamente significativa al comparar los resultados de estas pruebas. Los que asistieron en seis ocasiones o más a los laboratorios, demostraron un aumento significativo en las destrezas trabajadas en los cursos de lectura, escritura y matemáticas.

Por su parte, Langtry, Coupland y Moore (2003) realizaron una investigación durante cinco semestres con estudiantes que estaban matriculados en cursos subgraduados de Matemática; en estos, se realizó lo que los autores llaman una innovación en el proceso de enseñanza: integrar ejercicios de laboratorio incorporando el programado Mathematica $^{\mathrm{TM}}$. Los ejercicios expusieron a los estudiantes a una amplia variedad de situaciones reales en las que los problemas se describían y analizaban paso a paso usando este programado. En los análisis realizados se encontró que los estudiantes tuvieron experiencias positivas con el programa debido a que: 1) trabajaron la matemática como un todo y no fragmentada, 2) percibieron que tenían un ambiente de apoyo en los laboratorios de computadoras, y 3) pudieron corregir sus propios errores, entre 
otros. Ciertamente, los laboratorios llenaron las expectativas para las cuales fueron creados.

Por otro lado, Wilhelm y Walters (2006) investigaron la incorporación de laboratorios en los que se fomentaba el inquirir, tanto en un curso de Física introductoria como en cursos de metodología de la enseñanza de ciencia y de matemática. En los laboratorios, los estudiantes tuvieron la oportunidad de construir nuevos conocimientos, presentaron lo que habían aprendido a través de dibujos, narraciones, entrevistas, vídeos y representaciones gráficas. Visualizaron la relación que existía entre la teoría y el mundo real, tanto en ciencia como en matemática. Demostraron haber adquirido el conocimiento de los temas trabajados en el curso, ya que lo hicieron de forma aplicada. Los autores indican que los resultados de su investigación arrojan evidencia de la efectividad de este tipo de laboratorio y recomiendan la incorporación de los mismos.

Di Muro y Terry (2007) recomiendan incorporar los laboratorios en las clases de Matemática. Específicamente señalan que en estos se debe integrar asignaciones tanto individuales como en grupo, ya que el trabajo grupal los expone a múltiples experiencias de aprendizaje. Por ejemplo, les permite discutir posibilidades, comparar respuestas, interpretar y reflexionar sobre lo que aprenden. Recomiendan que los instructores fomenten los grupos de estudio, de modo que se puedan reunir para discutir las preguntas formuladas en los laboratorios y relacionarlas con el material discutido en los cursos. Indican que estos grupos deben ser heterogéneos, esto es, que los mismos estén constituidos por estudiantes que posean distintos estilos de aprendizaje, de manera que se compartan las fortalezas de cada uno.

Por su parte, Villareal (2003) realizó una investigación en la cual integró laboratorios; unos comprendían el uso de la computadora, en otros se ofrecía utilizar esta herramienta. El estudio se realizó con las clases de Álgebra de un colegio universitario del estado de Tejas. Los laboratorios fueron exitosos y su éxito se debió a las distintas modalidades utilizadas. La investigadora menciona que esta diferencia respondió a lo que indica la literatura, respecto al hecho de que los estudiantes aprenden de formas diversas, es decir, tienen 
distintos estilos de aprendizaje. Su trabajo encontró que aumentó la participación activa de los estudiantes tanto en las clases como en los laboratorios.

También, Litowitz (2009) señala que cuando los estudiantes aprenden matemática en laboratorios que integran la tecnología, la aprenden en un contexto invaluable de aprender haciendo, aspecto que les será de mucha utilidad en sus vidas. Además, la integración de la tecnología en la enseñanza de conceptos matemáticos, en particular de las calculadoras gráficas, es altamente recomendada por los investigadores.

En resumen, la literatura indica que el incorporar laboratorios en la clase de Matemática, redunda en un aumento en el aprovechamiento de los estudiantes (Fullmer, 2011). También se indica que los programas académicos exitosos utilizan múltiples estrategias de enseñanza- aprendizaje para mejorar y fortalecer el éxito de los estudiantes en los cursos de Matemática. Esto incluye el uso de la tecnología como un suplemento a la instrucción ofrecida en clases y la integración de los laboratorios en la instrucción (Bonham \& Boylan, 2011). También Villareal (2003) encontró que el incorporar estos en los cursos de Matemática fue efectivo ya que, entre otras cosas, fomentó la participación activa de los estudiantes tanto en las clases como en los laboratorios. Wilhelm y Walters (2006) también recomiendan la incorporación de laboratorios en los que se utilice el método de inquirir, ya que obtuvieron resultados positivos al utilizarlos con los estudiantes. Además, Shelly (2002) indica que los laboratorios propician una participación activa por parte de los alumnos. De igual forma, Di Muro y Terry (2007) recomiendan incorporar los laboratorios en las clases de Matemática, puesto que los mismos promueven el trabajo en grupo y permiten a los estudiantes discutir posibilidades, comparar respuestas e interpretar y reflexionar sobre lo que aprenden. También, Langtry, Coupland y Moore (2003) recomiendan el utilizar los laboratorios en las clases de Matemática, ya que los mismos redundaron en experiencias positivas para los alumnos. Estos les permitieron corregir sus propios errores y tener un ambiente de apoyo. También De Mestre (2005) recomienda incorporar los mismos en todos los niveles. 
Además, los laboratorios se han integrado en otras disciplinas como lo es la Ingeniería Mecánica (Tinnirello, Gago, \& Dadamo, 2010) y las ciencias (Koleza \& 2008). Estos también han resultado en un impacto positivo para los estudiantes. Finalmente, la integración de la tecnología en el proceso de enseñanza-aprendizaje de Matemática, en particular de las calculadoras gráficas, es altamente recomendado por los investigadores (Wee Leng, 2011).

\section{Metodología}

\section{Diseño del estudio}

Esta es una investigación de naturaleza cuantitativa. Para analizar los datos se realizó un análisis univariado: análisis de covarianza (ANCOVA) para comparar el rendimiento del examen de mitad de curso y el examen final, entre los estudiantes de todas las concentraciones que asistieron al laboratorio frente a los que no asistieron (Montgomery, 2012). Además, se realizó la misma comparación usando el factor concentración del estudiante, el cual tiene dos niveles: Contabilidad versus las otras concentraciones ofrecidas por la FAE. Se consideró esta comparación debido a que la concentración que más estudiantes tiene matriculados la FAE es Contabilidad.

\section{Participantes}

En este estudio participaron estudiantes que estaban matriculados en el curso MECU 3031 de la FAE durante el primer semestre 2013-2014. Participaron en total 137 estudiantes; estos formaron parte de alguna de las cinco secciones del curso, las cuales incluían estudiantes de las siguientes concentraciones de la FAE: Finanzas, Economía, Contabilidad, Sistemas Computarizados de Información, Gerencia de Operaciones, Mercadeo, Programa General y Recursos Humanos.

El contenido MECU 3031 es en su mayoría el de un curso de precálculo con aplicaciones a la administración de empresas. Se estudian las ecuaciones lineales en dos variables (gráficas, pendiente 
de la recta y aplicaciones); los sistemas de ecuaciones lineales en dos variables (diferentes métodos de solución y sus aplicaciones); matrices (definiciones básicas, operaciones, solución de sistemas de ecuaciones por el método de reducción); y programación lineal. Se da énfasis al concepto de función y se estudian las funciones lineales, cuadráticas, racional, raíz cuadrada, valor absoluto, partida, exponencial y logarítmica (dominio, alcance, evaluación, interceptos, gráfica y aplicaciones a diferentes áreas de la administración de empresas).

El estudiante tiene que aprobar el curso con una calificación como mínimo de C para poder matricularse en el curso que le sigue, esto es, Métodos Cuantitativos para Administración de Empresas II (MECU 3032). Para poder graduarse de la FAE, todo estudiante tiene que haber aprobado los cursos MECU 3031 y 3032, sin importar su concentración. Es importante destacar que el curso MECU 3031 es uno de los que se considera a nivel de la UPRRP, que provee las destrezas de razonamiento lógico matemático y destrezas necesarias para todos los estudiantes de Administración de Empresas, independiente de su concentración.

\section{Actividades}

Atodoslos estudiantes matriculados en MECU 3031 y participantes de la investigación se les requirió el uso de la calculadora gráfica en todo momento (en el salón de clases, en el laboratorio, en las asignaciones y en los exámenes). El curso se desarrolló mediante conferencias, ilustraciones, discusión de ejemplos, práctica de ejercicios y solución de problemas de aplicación en las áreas de Administración de Empresas. Durante la clase se fomentó que los estudiantes exploraran con los conceptos matemáticos, analizaran y llegaran a conclusiones. A todos los estudiantes matriculados en el curso se les evaluó mediante dos exámenes parciales (no coordinados), asignaciones y dos exámenes coordinados (uno de mitad de curso y el otro final). Las clases fueron impartidas por tres profesores con doctorado.

El propósito de los laboratorios fue fortalecer el contenido estudiado en el curso, aclarar dudas y discutir ejercicios y problemas, 
además de integrar la tecnología, en especial, la calculadora gráfica. En algunas ocasiones, se discutieron ejercicios mediante debates. Los laboratorios fueron ofrecidos por un ayudante de cátedra que estaba realizando estudios doctorales.

Se ofrecieron cuatro secciones de laboratorios, de una hora y veinte minutos. Los estudiantes asistieron a estas un día a la semana durante todo el semestre de forma voluntaria. Quienes participaron en los laboratorios, obtuvieron el $18 \%$ de su nota en el curso. Los ejercicios y problemas que se trabajaron en el laboratorio estaban alineados con los temas que se discutieron en el curso durante la semana. Tanto el curso como los laboratorios fueron coordinados por una profesora del Instituto de Estadística y Sistemas Computadorizados de Información.

\section{DesCRIPCión DE LOS DATOS}

En la investigación participaron estudiantes de las siguientes concentraciones: Finanzas, Economía, Contabilidad, Sistemas Computarizados de Información, Gerencia de Operaciones, Mercadeo, Programa General y Recursos Humanos. De cada participante del estudio se tomaron los siguientes datos: puntaje obtenido en el examen de mitad de curso, puntaje obtenido en el examen final y participación (asistencia y desempeño) en las clases de laboratorio. Entre los estudiantes que no asistieron al laboratorio del curso se encuentran los que libremente así lo decidieron y los que no lo completaron.

Además se recolectó la puntuación de Aprovechamiento Matemático en la Prueba de Evaluación y Admisión Universitaria que administra el College Board de Puerto Rico. La Tabla 2 presenta una descripción de los datos utilizados en este estudio. Se calculó la media, desviación estándar (S), mínimo, primer cuartil (Q1), mediana, tercer cuartil (Q3) y máximo; la variable participación en el laboratorio es categórica, se presentan la distribución de sus frecuencias. 
El EFECTO DEL LABORATORIO EN EL APROVECHAMIENTO DE LOS ESTUDIANTES...

Tabla 2

Descripción de los datos

\begin{tabular}{|c|c|c|c|c|c|c|c|}
\hline Variable & Media & $\mathrm{S}$ & Mínimo & Q1 & Mediana & Q3 & Máximo \\
\hline Aprov Mat & 643.31 & 74.26 & 438 & 590 & 661 & 689.5 & 787 \\
\hline Ex Mitad & 86.06 & 13.84 & 28 & 76 & 90 & 99.5 & 100 \\
\hline Ex Final & 66.28 & 17.83 & 20 & 55 & 68 & 80.0 & 97 \\
\hline \multicolumn{8}{|c|}{$\begin{array}{l}\text { Participación } \\
\text { en el laboratorio }\end{array}$} \\
\hline & Laboratorio & \multicolumn{2}{|c|}{ Frecuencia } & Porcentaje & & & \\
\hline & SÍ & \multicolumn{2}{|c|}{50} & 36.50 & & & \\
\hline & NO & \multicolumn{2}{|c|}{87} & 63.50 & & & \\
\hline & Total & \multicolumn{2}{|c|}{137} & 100.00 & & & \\
\hline
\end{tabular}

Fuente: Elaboración propia.

La Tabla 3 y Tabla 4 muestran el desempeño de los estudiantes en el examen de mitad de curso y el examen final, respectivamente.

Tabla 3

Examen de mitad de curso

\begin{tabular}{lcc}
\hline & NO lab. & Ś́ lab. \\
\cline { 2 - 3 } Media & 84.70 & 88.42 \\
Mediana & 88.00 & 92.00 \\
$\mathrm{~S}$ & 14.98 & 11.36 \\
$\mathrm{cv}$ & 17.69 & 12.85 \\
\hline
\end{tabular}

Fuente: Elaboración propia. 
Tabla 4

\begin{tabular}{lcc} 
Examen final & & \\
\hline \multirow{2}{*}{ Media } & NO lab. & SÍ lab. \\
\cline { 2 - 3 } Mediana & 64.48 & 69.42 \\
S & 66.67 & 70.00 \\
cv & 18.95 & 15.39 \\
& 29.39 & 22.17 \\
\hline
\end{tabular}

Fuente: Elaboración propia.

De los resultados obtenidos, se observa un mejor rendimiento promedio, así como notas más homogéneas (menor coeficiente de variabilidad, cv) entre los estudiantes que asistieron al laboratorio. Lo dicho anteriormente se confirma mediante el diagrama de cajas que se muestra en la Figura 1.
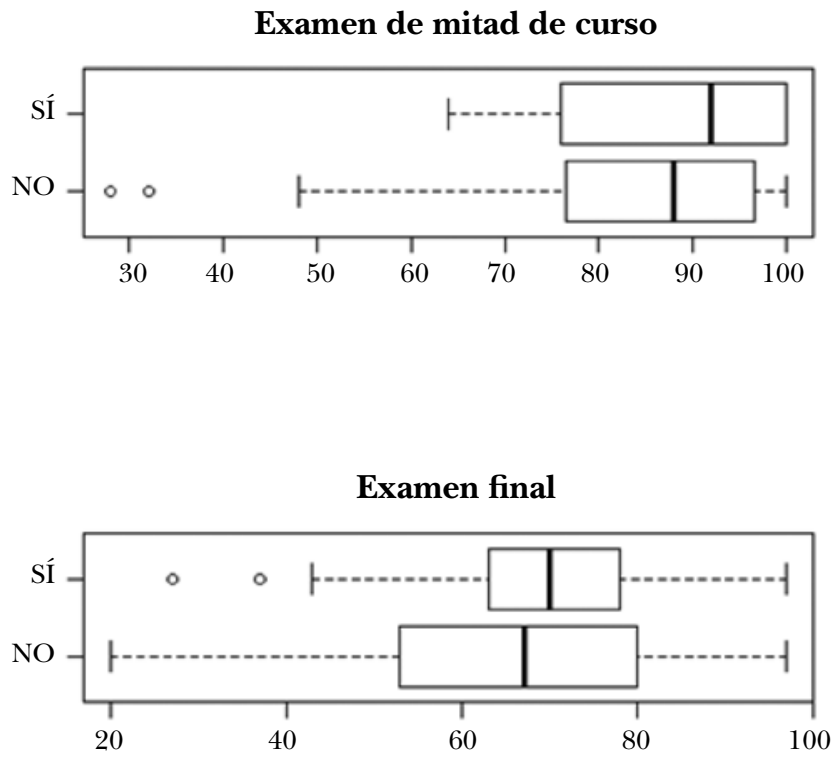

Figura 1. Variabilidad del examen de mitad de curso y examen final entre los estudiantes que Sí y NO asistieron al laboratorio. Elaboración propia. 
El EFECto del Laboratorio en El APROVECHAMIENTO DE LOS ESTUdiANTES...

\section{ANÁLISIS DE DATOS}

Análisis de un factor. Para la comparación de los resultados del examen de mitad de curso y del examen final, entre los estudiantes que SÍ y NO asistieron al laboratorio, se utilizó el análisis de covarianza (ANCOVA), usando como covariable los resultados del aprovechamiento matemático. El ANCOVA es una técnica estadística de comparación de medias, que combina el análisis de varianza (ANOVA) y el análisis de regresión. Mediante el ANCOVA se determinó la influencia de la covariable, es decir, el aprovechamiento matemático sobre la variable respuesta, esto es, el examen de mitad de curso o examen final, con el fin de eliminar dicha influencia y obtener una variable respuesta ajustada ( $\sin$ la influencia de la covariable), que se usará como criterio para comparar los niveles SÍ o NO del factor bajo estudio: la participación en el laboratorio.

Para una buena aplicación del ANCOVA fue importante verificar que no existen diferencias significativas en los promedios de grupos formados por los niveles del factor bajo estudio respecto a la covariable. En este estudio el factor es participación en el laboratorio con dos niveles: SÍ y NO; la covariable en estudio es el aprovechamiento matemático. Un análisis de estos datos muestra que efectivamente no hay diferencia ( pvalue $=0.266$ ) entre los promedios de aprovechamiento matemático entre los que Sí y NO asistieron al laboratorio.

En la Tabla 5, se verifica que el aprovechamiento matemático ha influido sobre los resultados del examen de mitad de curso y del examen final (pvalue $=0.000$ en ambos casos); por lo tanto, el análisis de covarianza está justificado.

Tabla 5

Examen de medio curso y examen final entre los que SÍ y NO asistieron al laboratorio

\begin{tabular}{lccccc}
\hline & & \multicolumn{2}{c}{ Examen de mitad de curso } & \multicolumn{2}{c}{ Examen final } \\
\cline { 3 - 6 } Fuentes & GL & F & Pvalue & F & Pvalue \\
Aprov Mat & 1 & 31.59 & 0.000 & 17.31 & 0.000 \\
Laboratorio & 1 & 4.90 & 0.028 & 4.21 & 0.042 \\
Error & 134 & & & & \\
Total & 136 & & & & \\
\hline
\end{tabular}

Fuente: Elaboración propia. 
Se concluye que hay diferencias significativas en los promedios de examen de mitad de curso y del examen final entre los que Sí y NO asistieron al laboratorio ( $p$ value $=<0.05$, en ambos casos). Los promedios del examen de mitad de curso ajustados por la covariable fueron: 89.2 y 84.3 para los que sí asistieron y no asistieron al laboratorio, respectivamente. Los promedios del examen final, ajustados por la covariable fueron: 70.2 y 64.0 para los que SÍ asistieron y NO asistieron al laboratorio, respectivamente. En ambos análisis un mejor promedio fue alcanzado por los que asistieron al laboratorio.

Análisis de dos factores. Además del factor participación en el laboratorio, con sus niveles SÍ y NO, se estudió el factor concentración del estudiante con dos niveles: 1) Contabilidad y 2) demás concentraciones de la FAE, que incluyen Economía, Finanzas, Sistemas de Información, Gerencia de Operaciones, Mercadeo, Programa General y Recursos Humanos. En la Tabla 6, se observa que la interacción de los factores participación en el laboratorio y concentración, no es significativa para el examen de mitad de curso ni para el examen final.

Tabla 6

Examen de mitad de curso y examen final frente a dos factores: Participación en el laboratorio y concentración

\begin{tabular}{lccccc}
\hline & & \multicolumn{2}{c}{ Examen de mitad de curso } & \multicolumn{2}{c}{ Examen final } \\
\cline { 3 - 6 } Fuentes & GL & F & Pvalue & F & Pvalue \\
Aprov Mat & 1 & 21.09 & 0.000 & 10.33 & 0.000 \\
Laboratorio & 1 & 5.49 & 0.021 & 6.19 & 0.014 \\
Concentración & 1 & 6.27 & 0.014 & 1.50 & 0.224 \\
Lab×Concentración & 1 & 0.58 & 0.450 & 0.04 & 0.836 \\
Error & 107 & & & & \\
Total & 111 & & & & \\
\hline
\end{tabular}

Fuente: Elaboración propia.

Por lo tanto, de los hallazgos, se puede afirmar que:

1) Hay diferencias significativas, en los promedios de examen de mitad de curso y del examen final entre los estudiantes de todas las concentraciones que Sí y NO asistieron al 
laboratorio (pvalue $<0.05$, en ambos casos). En ambos análisis, un mejor promedio fue alcanzado por los que asistieron al laboratorio.

2) Entre los estudiantes que SÍ asistieron al laboratorio (o entre los que NO asistieron al laboratorio) no hay diferencias significativas, en los promedios de los grupos de concentración: Contabilidad y los demás, respecto a las variables examen de mitad de curso o examen final. Además, se encontró que en los de Contabilidad (o en los de otras concentraciones de la FAE), no hay diferencias significativas entre los que SÍ y NO participaron del laboratorio, respecto a las variables examen de mitad de curso o examen final.

$\mathrm{Al}$ analizar las notas del examen de mitad de curso respecto al factor participación en el laboratorio (Tabla 6), se probó que el mejor promedio lo obtuvieron los estudiantes que Sí participaron de dicho laboratorio, sin importar su concentración. También respecto al factor concentración, se probó que el mejor promedio lo obtuvieron los estudiantes de Contabilidad. Al analizar las notas del examen final respecto al factor participación en el laboratorio, se probó que el mejor promedio lo obtuvieron los estudiantes que SÍ participaron de dicho laboratorio. Respecto al factor concentración, se probó que no hay diferencias significativas entre los promedios del examen final de estudiantes de Contabilidad frente a los promedios del examen final de estudiantes de las demás concentraciones de la FAE.

\section{Conclusiones y ReCOMENDACIONES}

De los hallazgos del estudio se desprende la efectividad de los laboratorios en el aprovechamiento de los estudiantes en el curso MECU 3031. Se obtuvo un mejor promedio en los estudiantes de todas las concentraciones que asistieron al laboratorio versus los que no asistieron, tanto en el examen de mitad de curso como en el examen final. Asimismo, para el examen de mitad de curso 
se obtuvo un mejor promedio en los estudiantes de Contabilidad versus los estudiantes de las demás concentraciones de la FAE. En cuanto a los resultados del examen final, no hubo diferencias entre los estudiantes de los dos grupos de concentraciones señaladas.

Estos resultados abonan a lo que establece la literatura en cuanto a la inclusión de laboratorios en diferentes disciplinas. Los programas exitosos son aquellos que usan múltiples estrategias de enseñanza. El hecho del gran porcentaje de fracasos de los estudiantes en los cursos cuantitativos está forzando a que se revalúe no solo los temas que se enseñan, sino las estrategias de enseñanza-aprendizaje que se utilizan, en todos los niveles, incluyendo el universitario.

Es altamente recomendable que se utilicen los laboratorios en los cursos, para que los estudiantes puedan: aclarar dudas, realizar ejercicios y problemas aplicados asistidos por la tecnología — como en el caso del estudio-, así como trabajar individualmente y en equipo. Es necesario también que la administración apoye estas iniciativas y provea asistentes de laboratorio y los recursos necesarios.

Se recomienda que se realicen otras investigaciones en las que se estudie el efecto de incorporar los laboratorios en los otros cursos de Métodos Cuantitativos y cursos subsiguientes. En particular, el integrar los laboratorios al curso MECU 3001, sería de gran utilidad para subsanar muchas de las dificultades que los estudiantes tienen, sobre todo en el área de álgebra elemental, de modo que adquieran las destrezas que necesitan para dominar los aspectos cuantitativos a los que se enfrentarán en su vida profesional. Además, les ayudará a adquirir las destrezas que aplicarán en cursos subsiguientes; por ejemplo, Contabilidad requiere destrezas de precálculo, mientras que Finanzas y Economía requieren destrezas más avanzadas de cálculo y probabilidad.

A pesar de que la calculadora gráfica incluye un programado matemático que permite diferentes representaciones de una función matemática —como ecuaciones, tablas de valores y gráficas—, también permite realizar operaciones con matrices y resolver sistemas de ecuaciones, entre otras. Se recomienda integrar en el laboratorio otros programados matemáticos, tales como: hojas electrónicas de cálculo, graficadores matemáticos disponibles de forma gratuita 
en internet y plataformas virtuales. Asimismo, en el laboratorio se debe fomentar que los estudiantes investiguen la aplicación de las matemáticas prácticas en la administración de empresas, para las diversas concentraciones.

Finalmente, se recomienda que se realicen futuras investigaciones relacionadas con el análisis de la efectividad de los laboratorios, de modo que sirvan de guía para tomar decisiones informadas relacionadas con los programas y sobre estrategias efectivas para mejorar el aprendizaje de los estudiantes en las diferentes disciplinas de la administración de empresas. El éxito de los estudiantes en los cursos cuantitativos redunda en beneficio de las universidades, ya que puede ayudar a aumentar la tasa de retención de los alumnos en estas.

El atender destrezas cuantitativas, como las que se trabajan en los cursos de Métodos Cuantitativos, desde que los estudiantes comienzan sus respectivas carreras universitarias, los ayudará a ser exitosos en cursos subsiguientes y a concluir su bachillerato en un menor periodo de tiempo (Vega Vilca \& Agosto, 2010). 


\section{RefERENCIAS}

American Mathematical Association of Two-Year Colleges. (2006). Beyond crossroads: Implementing mathematics standards in the first two years of college. Blair, R. (Ed.) Memphis, TN: Autor.

Aponte, G., Pagán, E., Quintero, A. H., Balet, S., Nazario, M., \& Velázquez, W. (2003). Múltiples escenarios de aprendizaje. Memorias del Congreso de Investigación sobre la Universidad, 166-187.

Bonham, B. S., \& Boylan, H. R. (2011). Developmental mathematics: Challenges, promising practices, and recent initiatives. Journal of Developmental Education, 34(3), 2-10.

Capraro, R. M., Capramo, M. M. \& Rupley, W. H. (2012). Readingenhanced word problem solving: A theoretical model. European Journal of Psychology of Education, 27(1), 91- 114.

De Mestre, N. (2005). Laboratory mathematics. Australian Mathematics Teacher, 61(1), 32-33.

Di Muro, P., \& Terry, M. (2007). A matter of style: Applying Kolb's learning style model to college mathematics teaching practices. Journal of College Reading and Learning, 38(1), 53-60.

Ediger, M. (2012). Quality teaching in mathematics. Education, 133(2), 235-238.

Fullmer, P. (2011). Assessment of tutoring laboratories in a learning assistance center. Journal of College Reading and Learning, 42(2), 67-89.

Gordon, S. (2010). The states of mathematics education today: What happens in the math classroom. Journal of Economics and Finance, 34, 471-476.

Kachapova, F., \& Kachapov, I. (2012). Percentage problems in bridging courses. International Journal of Mathematical Education in Science E् Technology, 43(5), 654-663.

Koleza, E., \& Pappas, J. (2008). The effect of motion analysis activities in a video-based laboratory in students' understanding of position, velocity and frames of reference. International Journal of Mathematics Education in Science and Technology, 39, 701-723.

Langtry, T., Coupland, M., \& Moore, B. (2003). Mathematica ${ }^{\mathrm{TM}}$ in context. International Journal of Mathematical Education in Science and Technology, 34, 699-718.

Montgomery, D. C. (2012). Design and analysis of experiments. (8a ed.). Hoboken, NJ: Wiley. 
National Council of Teachers of Mathematics. (2000). Principles and standards for school mathematics. Reston, VA: Autor.

National Council of Teachers of Mathematics. (2005). NCTM position paper on technology: The use of technology in the learning and teaching of mathematics. En W. J. Masalski \& P. C. Elliot (Eds.), Technology-supported mathematics learning environments; sixty-seventh yearbook (pp. 1-2). Reston, VA: Autor.

Litowitz, L. S. (2009). Addressing mathematics literacy through technology, innovation, design, and engineering. The Technology Teacher, 69, 19-22.

Senado Académico. Informe ejecutivo al Senado Académico sobre la Propuesta de Revisión del Componente Medular del bachillerato de la Facultad de Administración de Empresas, 2008. Río Piedras, PR: Universidad de Puerto Rico. Recuperado de http://senado. uprrp.edu/RevisionBA/Propuestas2007-08/IE2008-98-AECompMedular.pdf

Shelly, R. (2002). Designing math labs to accommodate advanced standards and technology. National Association of Secondary School Principals, NASSP Bulletin, 86, 45-58.

Tinnirello, A. M., Gago, E. A., \& Dadamo, M. B. (2010). Designing interdisciplinary interactive work: Basic sciences in engineering education. The International Journal of Interdisciplinary Social Sciences, 5, 331-334.

Vega Vilca, J. C. \& Agosto, F. (2010). Predicción del aprovechamiento académico: Estudio de los egresados de la Facultad de Administración de Empresas, Universidad de Puerto Rico, Recinto de Río Piedras. Revista de Ciencias Sociales, 23, 130-143.

Villareal, L. M. (2003). A step in the positive direction: Integrating a computer laboratory component into developmental algebra courses. Mathematics and Computer Education, 37, 72-78.

Wee Leng, N. (2011). Using an advanced graphing calculator in the teaching and learning of calculus. International Journal of Mathematics Education in Science and Technology, 42, 925-938.

Wilhelm, J. A., \& Walters, K. L. (2006). Pre-service mathematics teachers become full participants in inquiry investigations. International Journal of Mathematics Education, 37, 793-804.

Wiske, S. (2004). Use technology to dig for meaning. Educational Leadership, 62(1), 46-50. 